\title{
BMJ Open Comparison of conventional, doppler and contrast-enhanced ultrasonography in differential diagnosis of ovarian masses: a systematic review and meta- analysis
}

\author{
Lizhang Xun (D) , ${ }^{1}$ Lamei Zhai, ${ }^{2}$ Hui Xu ${ }^{1}$
}

To cite: Xun L, Zhai L, Xu H. Comparison of conventional, doppler and contrast-enhanced ultrasonography in differential diagnosis of ovarian masses: a systematic review and meta-analysis. BMJ Open 2021;11:e052830. doi:10.1136/ bmjopen-2021-052830

- Prepublication history and additional supplemental material for this paper are available online. To view these files, please visit the journal online (http://dx.doi.org/10.1136/ bmjopen-2021-052830).

LX and LZ contributed equally.

Received 06 May 2021 Accepted 18 November 2021

Check for updates

(c) Author(s) (or their employer(s)) 2021. Re-use permitted under CC BY-NC. No commercial re-use. See rights and permissions. Published by BMJ.

${ }^{1}$ Medical Examination Center, Huaian City Second People's Hospital, Huaian, Jiangsu, China ${ }^{2}$ Department of Radiology, Huaian City Second People's Hospital, Huaian, Jiangsu, China

Correspondence to

Dr Lizhang Xun;

7223198@qq.com

\section{ABSTRACT}

Objectives To assess the value of conventional, Doppler and contrast-enhanced ultrasonography (CEUS) (conventional ultrasonography (US), Doppler US and CEUS) for diagnosing ovarian cancer.

Design Systematic review and meta-analysis. Data sources PubMed, Embase and the Cochrane Library were conducted for studies published until October 2021.

Eligibility criteria Studies assessed the diagnostic value of conventional US, Doppler US or CEUS for detecting ovarian cancer, with no restrictions placed on published language and status.

Data extraction and synthesis The study selection and data extraction were performed by two independent authors. The sensitivity, specificity, positive and negative likelihood ratio (PLR and NLR), diagnostic OR (DOR) and area under the receiver operating characteristic curve (AUC) were pooled using the bivariate generalised linear mixed model and random effects model.

Results The meta-analysis included 72 studies and involved 9296 women who presented with ovarian masses. The pooled sensitivity, specificity, PLR, NLR, DOR and AUC for conventional US were 0.91 (95\% Cl: 0.87 to 0.94$)$ and 0.87 (95\% Cl: 0.82 to 0.91 ), 6.87 (95\% Cl: 4.98 to 9.49 ) and 0.10 (95\% Cl: 0.07 to 0.15$), 57.52$ (95\% Cl: 36.64 to $90.28)$ and 0.95 (95\% Cl: 0.93 to 0.97$)$, respectively. The sensitivity, specificity, PLR, NLR, DOR and AUC for Doppler US were 0.93 (95\% Cl: 0.91 to 0.95$)$ and $0.85(95 \% \mathrm{Cl}$ : 0.80 to 0.89 ), 6.10 (95\% Cl: 4.59 to 8.11$)$ and $0.08(95 \%$ Cl: 0.06 to 0.11$), 61.76$ (95\% Cl: 39.99 to 95.37$)$ and 0.96 ( $95 \%$ Cl: 0.94 to 0.97 ), respectively. The pooled sensitivity, specificity, PLR, NLR, DOR and AUC for CEUS were 0.97 (95\% Cl: 0.92 to 0.99 ) and 0.92 (95\% Cl: 0.85 to 0.95 ), 11.47 (95\% Cl: 6.52 to 20.17$)$ and 0.03 (95\% Cl: 0.01 to 0.09), 152.11 (95\% Cl: 77.77 to 297.51 ) and $0.99(95 \%$ Cl: 0.97 to 0.99$)$, respectively. Moreover, the AUC values for conventional US ( $p=0.002)$ and Doppler US $(p=0.005)$ were inferior to those of CEUS.

Conclusions Conventional US, Doppler US and CEUS have a relatively high differential diagnostic value for differentiating between benign and malignant ovarian masses. The diagnostic performance of CEUS was superior to that of conventional US and Doppler US.
Strengths and limitations of this study

- This study provides indirect comparison analyses among conventional ultrasonography, Doppler ultrasonography and contrast-enhanced ultrasonography for detecting ovarian cancer.

- This study included prospective, retrospective and cross-sectional studies; moreover, the results could be affected by uncontrolled selective and recall biases.

- Subgroup analyses according to country and route were performed.

- Inevitable publication bias and restricted detailed analyses are limitations.

\section{INTRODUCTION}

Annually, an estimated 60000 women in the USA undergo surgical excisions for adnexal masses or suspected ovarian neoplasm; moreover, approximately 313959 ovarian cancer cases were diagnosed in 2020 worldwide. ${ }^{2}$ Adnexal masses are often incidentally observed given widespread diagnostic imaging use; further, most cases are diagnosed with benign masses. ${ }^{34}$ Currently, most newly diagnosed ovarian cancer (OC) cases are at stages III and IV, with the survival rate ranging from $25 \%$ to $30 \% .^{5}$ However, the survival rate for OC at stage I could be as high as $90 \% .{ }^{6}$ Therefore, early OC detection and accurate tumour property assessment remain important issues in clinical practice. ${ }^{7}$

Currently, there are no reliable approaches for early OC detection; however, early-stage differential diagnosis of benign and malignant ovarian masses is important. The use of ultrasonography (US) for determining benign or malignant ovarian masses is mainly based on subjective and qualitative diagnosis. The current overall diagnostic accuracy of US for OC could reach $80 \%{ }^{8}$ Conventional 
US can visualise the capsule and tumour shapes, which could allow differential diagnosis of benign or malignant tumours. ${ }^{9}$ Angiogenesis could be involved in tumour growth and metastasis; additionally, it is significantly correlated with malignant tumours. ${ }^{10}$ Moreover, spectral analysis of Doppler US could detect the blood flow status in tumours through the Doppler waveform. ${ }^{11}$ Furthermore, contrast-enhanced US (CEUS) could improve imaging quality. ${ }^{12}$ However, the diagnostic values of conventional US, Doppler US and CEUS for differentiating between benign and malignant ovarian masses have not been compared. Therefore, we aimed to perform a systematic review and meta-analysis to assess the value of conventional US, Doppler US and CEUS for differential diagnosis of benign and malignant ovarian masses. Moreover, we aimed to perform indirect comparison analysis to compare the diagnostic value among conventional US, Doppler US and CEUS.

\section{METHODS}

\section{Data sources, search strategy and selection criteria}

This systematic review and meta-analysis was performed and reported following the Preferred Reporting Items for Systematic Reviews and Meta-Analysis statement. ${ }^{13}$ There were no restrictions regarding publication language and status. Studies assessing the diagnostic value of conventional US, Doppler US or CEUS differentiating between benign and malignant ovarian masses were considered eligible for our analysis. We systematically searched PubMed, Embase and the Cochrane Library for eligible studies published until October 2021. The following search terms were used as text words or Medical Subject Heading terms: "ovarian neoplasms" AND ("ultrasonography" OR "Doppler ultrasonography" OR "contrastenhanced ultrasonography") AND "diagnosis." In addition, we manually reviewed the reference lists of the retrieved studies to identify new eligible studies.

Two authors (LX and LZ) independently performed the literature search and study selection, with disagreements being resolved by group discussion after reading the full-text of available articles. The inclusion criteria were as follows: (1) Study design: no restrictions were placed on study design, including cross-sectional, retrospective and prospective design; (2) Participants: adult women experience ovarian masses; (3) Diagnostic tool: conventional US, Doppler US or CEUS; (4) Gold standard: pathological; and (5) Analysis data: true and false positive, as well as true and false negative for differentiating between benign and malignant ovarian masses.

\section{Data collection and quality assessment}

Two authors (LX and LZ) independently performed data collection and quality assessment. The following data were collected: first author's name, publication year, country, sample size (malignant/benign), age, type of OC, modality, route, agent, US machine, true and false positive and true and false negative. The Quality
Assessment of Diagnostic Accuracy Studies was applied to assess the methodological bias for individual study based on patient selection, index test, reference standard, risk of bias and concerns regarding applicability. ${ }^{14}$ Betweenauthor inconsistencies concerning data collection and quality assessment were settled by an additional author (HX) who reviewed the full-text of the original article.

\section{Statistical analysis}

We applied true and false positive and negative in each study to calculate the sensitivity, specificity, positive likelihood ratio (PLR), negative likelihood ratio (NLR), diagnostic OR (DOR) and area under the receiver operating characteristic curve (AUC). Subsequently, the pooled diagnostic effect estimates for conventional US, Doppler US and CEUS were calculated using the bivariate generalised linear mixed model and random effects model. ${ }^{15-17}$ Heterogeneity across the included studies was assessed using $\mathrm{I}^{2}$ and $\mathrm{Q}$ statistic, with $\mathrm{I}^{2}>50.0 \%$ or $\mathrm{p}<0.10$ indicating significant heterogeneity. ${ }^{18} 19$ Next, the diagnostic value for conventional US, Doppler US and CEUS was calculated using an indirect comparison approach. ${ }^{20} \mathrm{We}$ performed subgroup analysis for the diagnostic performance of conventional US, Doppler US and CEUS according to country and route; subsequently, betweensubgroup differences were assessed using the interaction $\mathrm{P}$ test. ${ }^{21}$ Moreover, publication biases for the diagnostic value of conventional US, Doppler US and CEUS were assessed using the funnel plot and Deeks' asymmetry test. ${ }^{22}$ The inspection level for pooled results was twosided, with $\mathrm{p}<0.05$ being considered statistically significant. All statistical analyses were performed using the software Stata (V.10.0; Stata Corporation).

\section{Patient and public involvement}

No patient involved.

\section{RESULTS}

\section{Literature search}

The initial electronic searches identified 4028 articles; among them, 3192 were retained after removing duplicate articles. Subsequently, 3038 studies were excluded for reporting irrelevant topics. The remaining 154 studies were retrieved for further full-text evaluations, with 82 studies being excluded for the following reasons: other diagnostic tools $(n=45)$, combined diagnostic strategies $(n=31)$ and insufficient data $(n=6)$. The remaining 72 studies were included in the final meta-analysis. No eligible study was identified from reviewing the reference lists of the included studies. Figure 1 presents the detailed results regarding the study selection.

\section{Study characteristics}

The characteristics of the identified studies and recruited patients are shown in online supplemental 1 . The included studies involved 9296 women presenting ovarian masses, with the sample size ranging from 19 to 826 . 


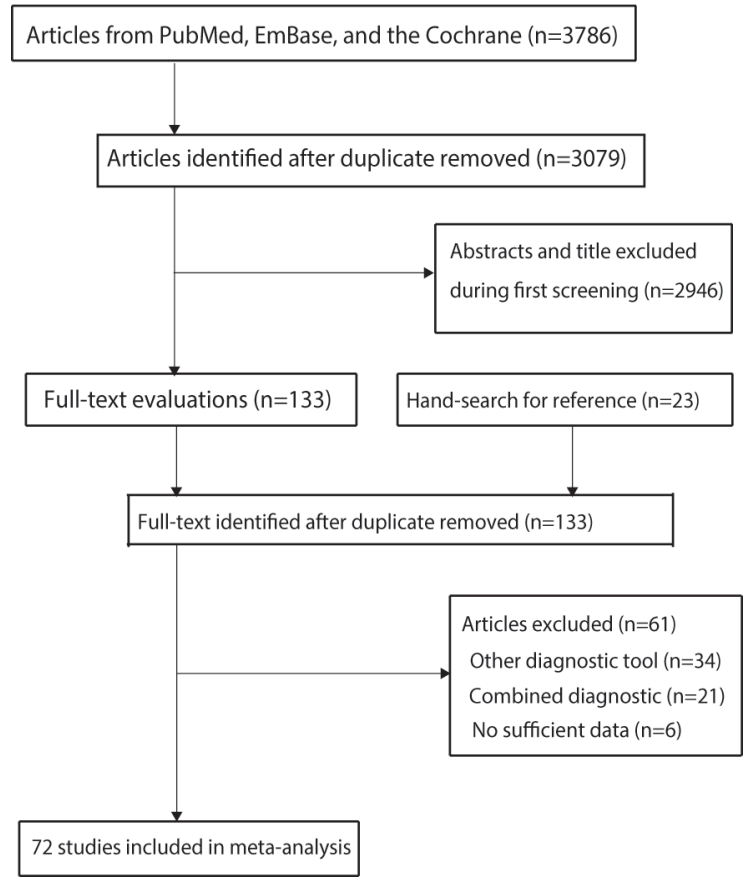

Figure 1 The Preferred Reporting Items for Systematic Reviews and Meta-Analyses flowchart for the study selection process.

Among the included studies, 24 were conducted in Asia with the remaining 48 studies being conducted in Europe or America. Further, 36, 51 and 29 cohorts assessed the diagnostic performance of conventional US, Doppler US and CEUS, respectively. Online supplemental 2 presents the details regarding the quality of each study with most of them having moderate-to-high quality.

\section{Sensitivity and specificity}

The pooled sensitivity and specificity for conventional US in the differential diagnosis of benign and malignant ovarian masses were 0.91 (95\% CI: 0.87 to 0.94 ) and 0.87 (95\% CI: 0.82 to 0.91 ), respectively. The values for pooled sensitivity and specificity in Doppler US were 0.93 (95\% CI: 0.91 to 0.95 ) and 0.85 (95\% CI: 0.80 to $0.89)$, respectively. Furthermore, the summary sensitivity and specificity for CEUS were 0.97 (95\% CI: 0.92 to 0.99 ) and 0.92 (95\% CI: 0.85 to 0.95 ), respectively (online supplemental 3). Conventional US had a lower sensitivity than CEUS for differentiating between benign and malignant ovarian masses (ratio: 0.94; 95\% CI: 0.89 to $0.99 ; \mathrm{p}=0.019$ ). Doppler US had a lower specificity than CEUS for differentiating between benign and malignant ovarian masses (ratio: $0.92 ; 95 \%$ CI: 0.86 to $1.00 ; \mathrm{p}=0.044$ ) (table 1). Subgroup analysis revealed high sensitivity of conventional US and Doppler US in the transvaginal group (table 2).

\section{PLR and NLR}

The pooled PLR and NLR for conventional US differentiating between benign and malignant ovarian masses were 6.87 (95\% CI: 4.98 to 9.49 ), and 0.10 (95\% CI: 0.07 to $0.15)$, respectively. The corresponding values for Doppler

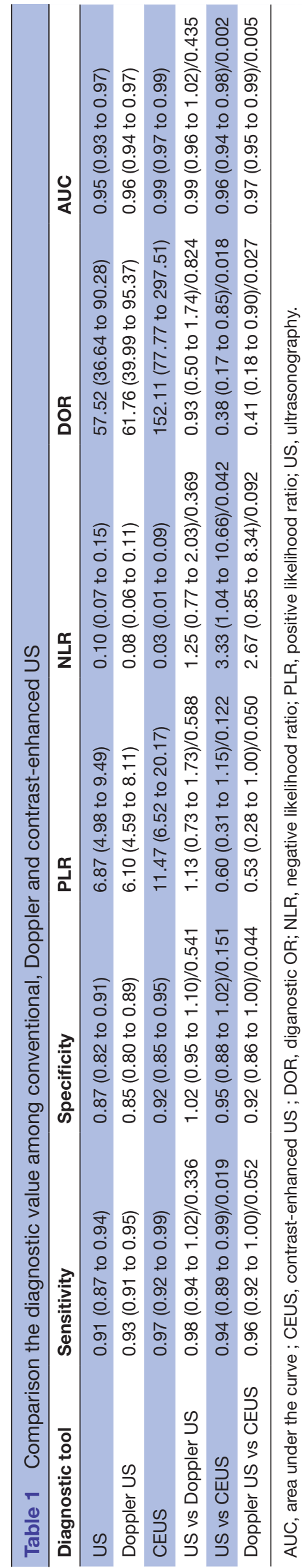




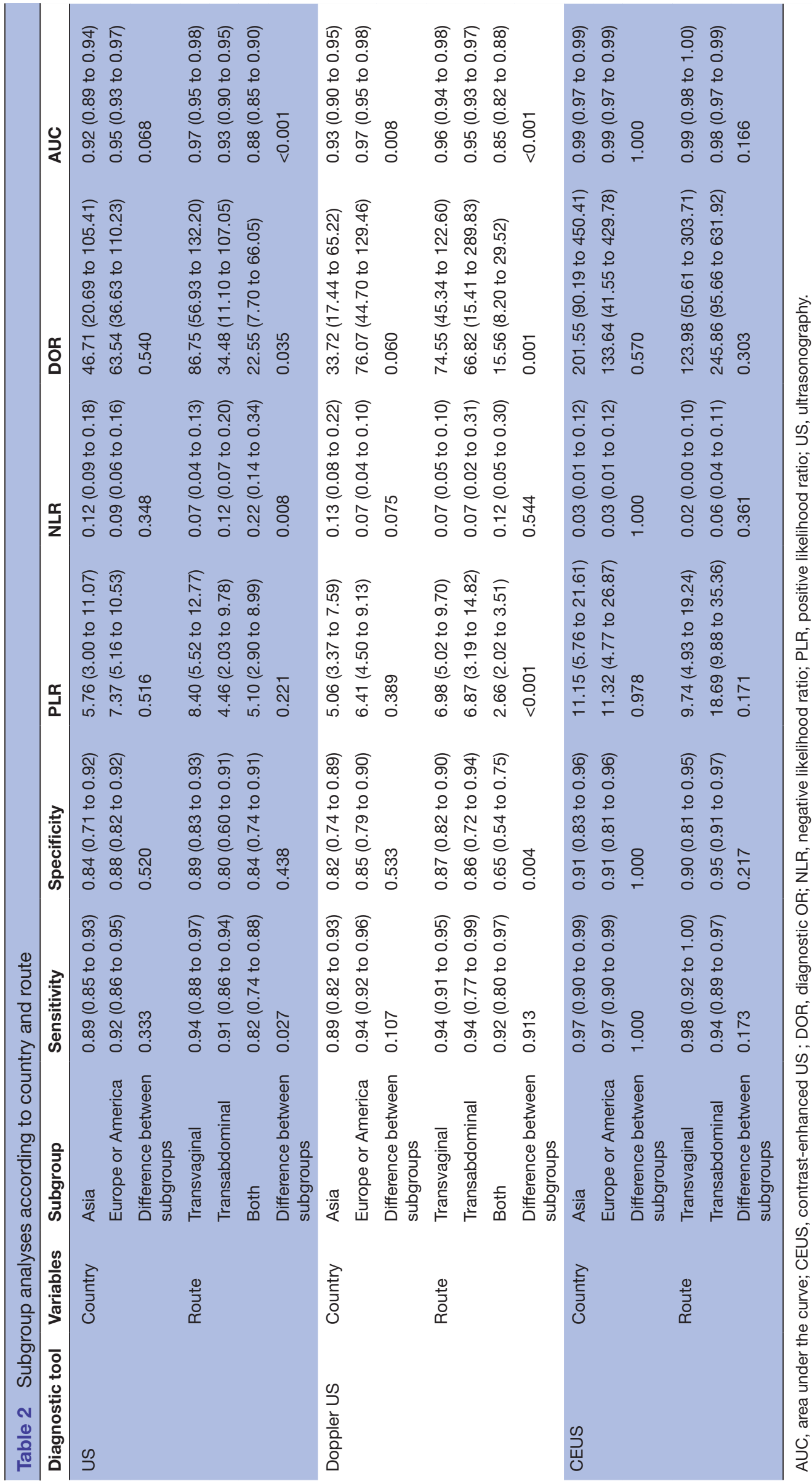




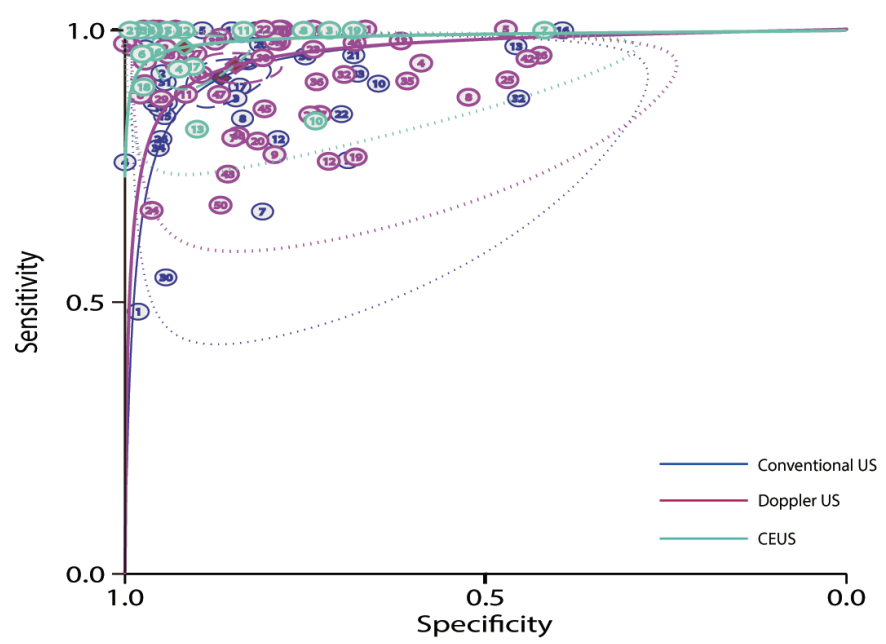

Figure 2 The area under the curve of conventional US, Doppler US and CEUS for differentiating between malignant and benign ovarian masses. CEUS, contrast-enhanced US; US, ultrasonography.

US were 6.10 (95\% CI: 4.59 to 8.11 ) and 0.08 (95\% CI: 0.06 to 0.11 ) for pooled PLR and NLR, respectively. Furthermore, the summary PLR and NLR for CEUS were 11.47 (95\% CI: 6.52 to 20.17 ) and 0.03 (95\% CI: 0.01 to 0.09 ), respectively (online supplemental 4 ). Conventional US versus CEUS showed higher NLR (ratio: 3.33; 95\% CI: 1.04 to 10.66 ; $\mathrm{p}=0.042$ ), while Doppler US versus CEUS showed lower PLR (ratio: 0.53; 95\% CI: 0.28 to 1.00; $\mathrm{p}=0.050$ ) (table 1). Subgroup analyses suggested that the NLR for conventional US and PLR for Doppler US were lower and higher in the transvaginal group, respectively (table 2).

\section{DOR}

The pooled DOR of conventional US, Doppler US and CEUS for differentiating between benign and malignant ovarian masses were 57.52 (95\% CI: 36.64 to 90.28), 61.76 (95\% CI: 39.99 to 95.37 ) and 152.11 (95\% CI: 77.77 to 297.51), respectively (online supplemental 5). There was significant heterogeneity across the included studies for conventional US $\left(\mathrm{I}^{2}=66.5 \% ; \mathrm{p}<0.001\right)$ and Doppler US $\left(I^{2}=73.9 \% ; p<0.001\right)$ but not for CEUS $\left(I^{2}=25.7 \%\right.$; $\mathrm{p}=0.147$ ). The DOR of conventional US (ratio: 0.38; 95\% CI: 0.17 to $0.85 ; \mathrm{p}=0.018$ ) and Doppler US (ratio: $0.41 ; 95 \%$ CI: 0.18 to $0.90 ; p=0.027$ ) were significantly lower than that of CEUS for differentiating between benign and malignant ovarian masses (table 1). Subgroup analysis revealed that the DOR was high for conventional US and Doppler US in the transvaginal group (table 2).

\section{AUC}

The AUC of conventional US, Doppler US and CEUS for differentiating between benign and malignant ovarian masses were 0.95 (95\% CI: 0.93 to 0.97 ), 0.96 (95\% CI: 0.94 to 0.97 ) and 0.99 (95\% CI: 0.97 to 0.99 ), respectively (figure 2). Compared with CEUS, conventional US (ratio: 0.96 ; $95 \%$ CI: 0.94 to $0.98 ; \mathrm{p}=0.002$ ) and Doppler US (ratio: $0.97 ; 95 \%$ CI: 0.95 to 0.99 ; $p=0.005$ ) had significantly lower AUC values for detecting OC (table 1). Subgroup analysis suggested that the AUC of conventional US was affected by route and that the diagnostic value was high in the transvaginal group. Moreover, the AUC of Doppler US could be affected by country and route; further, the diagnostic value was high in the study groups from Europe or America, as well as in the transvaginal group (table 2 ).

\section{Publication bias}

Publication bias was also tested for in the diagnostic performance of conventional US, Doppler US and CEUS (figure 3). There were potentially significant publication biases for conventional US $(\mathrm{p}=0.02)$, Doppler US $(\mathrm{p}=0.04)$ and CEUS $(\mathrm{p}=0.02)$. However, after adjusting for potential publication bias, the diagnostic performance remained stable. ${ }^{23}$

\section{DISCUSSION}

The current systematic review and meta-analysis assessed the diagnostic performance of conventional US, Doppler

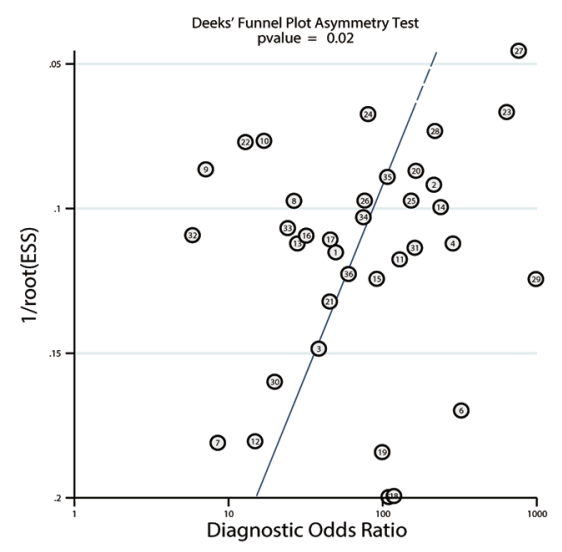

A. Conventional US

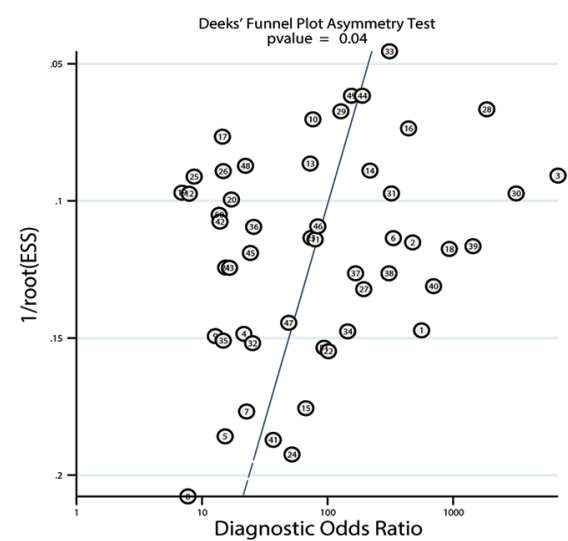

B. Doppler US

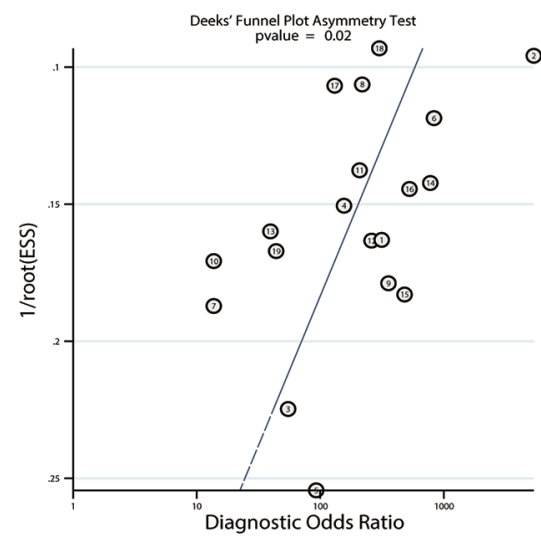

C. CEUS

Figure 3 Publication biases for conventional US, Doppler US and CEUS. CEUS, contrast-enhanced US; US, ultrasonography. 
US and CEUS for differentiating between benign and malignant ovarian masses. This comprehensive, largescale quantitative analysis included 9296women with diverse individual characteristics assessed in 72 studies. There was a relatively high diagnostic value of conventional US, Doppler US and CEUS for differentiating between benign and malignant ovarian masses. Moreover, indirect comparison analysis revealed that the diagnostic value of CEUS was superior to that of conventional US and Doppler US. Moreover, there was a significant difference in the diagnostic performance between conventional US and Doppler US. Subgroup analysis suggested that the diagnostic value of conventional US could be affected by route, while country and route could affect the diagnostic performance of Doppler US.

There have been several systematic reviews and metaanalyses on the diagnostic performance of conventional US, Doppler US and CEUS for detecting OC. Medeiros et al found that the colour Doppler US could be a useful preoperative tool for diagnosing OC from pelvic masses. ${ }^{24}$ Several studies also found CEUS had a high diagnostic value for differentiating between malignant and benign ovarian masses. ${ }^{25-27} \mathrm{~A}$ meta-analysis conducted by Liu et al on 67 high-quality studies suggested that conventional US, Doppler US and CEUS had a relatively high diagnostic value for OC. ${ }^{28}$ However, the aforementioned studies only reported the pooled diagnostic performance of conventional US, Doppler US and CEUS for differentiating between benign and malignant ovarian masses. Specifically, they did not compare among conventional US, Doppler US and CEUS; further, they did not illustrate the diagnostic performance of conventional US, Doppler US and CEUS based on country and route. Therefore, the current systematic review and meta-analysis assessed the diagnostic performance of conventional US, Doppler US and CEUS in differentiating between malignant and benign ovarian masses.

In the present study, there was a relatively high diagnostic performance of conventional US, Doppler US and CEUS for differentiating between benign and malignant ovarian masses, which is consistent with previous studies. ${ }^{24-28}$ A meta-analysis performed by Liu et al found similar diagnostic value among US, CT and MRI. ${ }^{8}$ Medeiros et al found the are under curve of MRI for detecting malignant OC was $0.9526,{ }^{29}$ which was similar compared with conventional US and Doppler US, but lower than CEUS from our study. Conventional US by placing a high frequency probe to scan the area adjacent to the sonic speed near field does not require a full bladder and is not affected by intestinal gas; moreover, it yields high-quality images. ${ }^{30}$ Subjective evaluation of the colour content of ovarian tumours through Doppler US is simple with low colour content indicating benignity. ${ }^{31}$ Moreover, the blood flow velocity in Doppler US could differentiate between benign and malignant pelvic masses. ${ }^{28}$ We observed similar diagnostic performance between conventional US and Doppler US for differentiating between benign and malignant ovarian masses; furthermore, the role of Doppler US could be affected by the resistance index; the use of Doppler US to assess the grey-scale ultrasound morphology in an adnexal mass with high accurate for predicting its nature. ${ }^{32}$ Moreover, CEUS had a higher diagnostic value than conventional US and Doppler US for differentiating between benign and malignant ovarian masses. This could be attributed to contrast agent injection improving the map of vascular anatomy, as well as the detection of signals from blood vessels with a diameter of $<40 \mu \mathrm{m}$. Therefore, CEUS could effectively visualise a greater vessel number in malignant than in benign tumours. ${ }^{33}$ Finally, the time-intensity curve parameters applied quantitatively assessed the kinetics of contrast agents in tumours, which was objective and reproducible and could be used for inexperienced examiners. ${ }^{35}$

In the present study, subgroup analyses revealed that route could affect the diagnostic performance of conventional US while country and route could affect the diagnostic performance of Doppler US for differentiating between benign and malignant ovarian masses. The aforementioned findings could be attributed to several reasons: (1) the number of studies in each subgroup was imbalanced and there were variable diagnostic performances of conventional US, Doppler US and CEUS; (2) there was between-study inconsistency in the prestudy US training, which could affect the diagnostic performance of conventional US, Doppler US and CEUS for differentiating between benign and malignant ovarian masses; and (3) most of the included studies performed transvaginal US with power stability, with fewer studies applying transabdominal US or both transvaginal and transabdominal US. Future large-scale prospective studies should verify these results.

This study has the following strengths: (1) the analysis was based on a large number of published studies and a large sample size, and therefore our findings are more robust than those of any individual study; (2) indirect comparison analyses were conducted to compare the diagnostic performance among conventional US, Doppler US and CEUS for differentiating between benign and malignant ovarian masses; and (3) stratified analyses for the diagnostic performance of conventional US, Doppler US and CEUS were conducted according to country and route, which allowed assessment of the diagnostic value in specific subpopulations.

Nonetheless, this study has several limitations. First, this study included prospective, retrospective and crosssectional studies; moreover, the results could be affected by uncontrolled selective and recall biases. Second, the experience levels of clinicians in US could have differed, which could affect the diagnostic performance of conventional US, Doppler US and CEUS. Third, the agents used for CEUS differed across the included studies, which could induce heterogeneity in the diagnostic value of CEUS. Fourth, the type of ovarian mass could affect the diagnostic performance of conventional US, Doppler US and CEUS, while the stratified data according to ovarian 
mass type were not available. Fifth, we performed an indirect comparison of diagnostic performance among conventional US, Doppler US and CEUS. Finally, there are inherent limitations of meta-analysis based on published articles, including the use of pooled data for analysis and the inevitable publication bias.

\section{CONCLUSION}

We observed a relatively high diagnostic performance of conventional US, Doppler US and CEUS for differentiating between malignant and benign ovarian masses. Moreover, the diagnostic value of CEUS was higher than that of conventional US and Doppler US. Furthermore, the diagnostic performance of conventional US could be affected by route, while country and route could affect the diagnostic value of Doppler US. Further large-scale prospective studies should directly compare the diagnostic performance of conventional US, Doppler US and CEUS for diagnosing OC.

Contributors LZ came up with the research idea and completed the study design. HX contributed to paper inclusion and data analysis. LX wrote the first draft of manuscript and finalised it with LZ. LX approved the submission of the final version of this paper. LX acts as a gaurantor for this study.

Funding The authors have not declared a specific grant for this research from any funding agency in the public, commercial or not-for-profit sectors.

Competing interests None declared.

Patient consent for publication Not applicable.

Ethics approval This study does not involve human participants.

Provenance and peer review Not commissioned; externally peer reviewed.

Data availability statement All data relevant to the study are included in the article or uploaded as supplementary information. The data sets generated and analysed during the current study are all presented in the manuscript, and additional materials are available from the corresponding author on reasonable request.

Supplemental material This content has been supplied by the author(s). It has not been vetted by BMJ Publishing Group Limited (BMJ) and may not have been peer-reviewed. Any opinions or recommendations discussed are solely those of the author(s) and are not endorsed by BMJ. BMJ disclaims all liability and responsibility arising from any reliance placed on the content. Where the content includes any translated material, BMJ does not warrant the accuracy and reliability of the translations (including but not limited to local regulations, clinical guidelines, terminology, drug names and drug dosages), and is not responsible for any error and/or omissions arising from translation and adaptation or otherwise.

Open access This is an open access article distributed in accordance with the Creative Commons Attribution Non Commercial (CC BY-NC 4.0) license, which permits others to distribute, remix, adapt, build upon this work non-commercially, and license their derivative works on different terms, provided the original work is properly cited, appropriate credit is given, any changes made indicated, and the use is non-commercial. See: http://creativecommons.org/licenses/by-nc/4.0/.

ORCID iD

Lizhang Xun http://orcid.org/0000-0001-9032-3898

\section{REFERENCES}

1 Liu JH, Zanotti KM. Management of the adnexal mass. Obstet Gynecol 2011;117:1413-28.

2 Sung H, Ferlay J, Siegel RL, et al. Global cancer statistics 2020: GLOBOCAN estimates of incidence and mortality worldwide for 36 cancers in 185 countries. CA Cancer J Clin 2021;71:209-49.

3 Menon U, Gentry-Maharaj A, Hallett R, et al. Sensitivity and specificity of multimodal and ultrasound screening for ovarian cancer, and stage distribution of detected cancers: results of the prevalence screen of the UK Collaborative trial of ovarian cancer screening (UKCTOCS). Lancet Oncol 2009;10:327-40.

4 Buys SS, Partridge E, Black A, et al. Effect of screening on ovarian cancer mortality: the prostate, lung, colorectal and ovarian (PLCO) cancer screening randomized controlled trial. JAMA 2011;305:2295.

5 Clarke-Pearson DL. Clinical practice. Screening for ovarian cancer. $N$ Engl J Med 2009;361:170-7.

6 Myers ER, Bastian LA, Havrilesky LJ. Management of adnexial mass. Evid Rep Technol Assess 2006:1-145.

7 Lutz AM, Willmann JK, Drescher CW, et al. Early diagnosis of ovarian carcinoma: is a solution in sight? Radiology 2011;259:329-45.

8 Liu J, Xu Y, Wang J. Ultrasonography, computed tomography and magnetic resonance imaging for diagnosis of ovarian carcinoma. Eur J Radiol 2007;62:328-34.

9 Sinnett V, Chavaria J, Downey K. Well-documented benign sonographic characteristics are rarely seen in malignant masses: is now the time to biopsy less? Clin Radiol 2021;76:787.e9-787.e13.

10 Hicklin DJ, Ellis LM. Role of the vascular endothelial growth factor pathway in tumor growth and angiogenesis. J Clin Oncol 2005;23:1011-27.

11 Delorme S, Knopp MV. Non-invasive vascular imaging: assessing tumour vascularity. Eur Radiol 1998;8:517-27.

12 Sidhu PS, Cantisani V, Dietrich CF, et al. The EFSUMB guidelines and recommendations for the clinical practice of contrast-enhanced ultrasound (CEUS) in non-hepatic applications: update 2017 (long version). Ultraschall Med 2018;39:e2-44.

13 Page MJ, McKenzie JE, Bossuyt PM, et al. The PRISMA 2020 statement: an updated guideline for reporting systematic reviews. BMJ 2021;372:n71.

14 Whiting PF, Rutjes AWS, Westwood ME, et al. QUADAS-2: a revised tool for the quality assessment of diagnostic accuracy studies. Ann Intern Med 2011;155:529-36.

15 DerSimonian R, Laird N. Meta-analysis in clinical trials. Control Clin Trials 1986;7:177-88.

16 Ades AE, Lu G, Higgins JPT. The interpretation of randomeffects meta-analysis in decision models. Med Decis Making 2005;25:646-54.

17 Walter SD. Properties of the summary receiver operating characteristic (SROC) curve for diagnostic test data. Stat Med 2002;21:1237-56.

18 Deeks JJ, Higgins JPT, Altman DG. Analyzing data and undertaking meta-analyses. In: Higgins J, Green S, eds. Cochrane Handbook for systematic reviews of interventions 5.0.1. Oxford, UK: The Cochrane Collaboration, 2008: chap 9 .

19 Higgins JPT, Thompson SG, Deeks JJ, et al. Measuring inconsistency in meta-analyses. BMJ 2003;327:557-60.

20 Woodward M. Epidemiology: study design and data analysis. Chapman \& Hall/CRC, 2000: 252-73.

21 Altman DG, Bland JM. Interaction revisited: the difference between two estimates. BMJ 2003;326:219.

22 Deeks JJ, Macaskill P, Irwig L. The performance of tests of publication bias and other sample size effects in systematic reviews of diagnostic test accuracy was assessed. J Clin Epidemiol 2005;58:882-93.

23 Duvall S. Tweedie R. A nonparametric "trim and fill" method for assessing publication bias in meta-analysis. J Am Stat Assoc 2000;95:89-98.

24 Medeiros LR, Rosa DD, da Rosa Ml, et al. Accuracy of ultrasonography with color Doppler in ovarian tumor: a systematic quantitative review. Int J Gynecol Cancer 2009;19:1214-20.

25 Wu Y, Peng H, Zhao X. Diagnostic performance of contrastenhanced ultrasound for ovarian cancer: a meta-analysis. Ultrasound Med Biol 2015;41:967-74.

26 Qiao J-J, Yu J, Yu Z, et al. Contrast-enhanced ultrasonography in differential diagnosis of benign and malignant ovarian tumors. PLOS One 2015;10:e0118872.

27 Ma X, Zhao Y, Zhang B, et al. Contrast-enhanced ultrasound for differential diagnosis of malignant and benign ovarian tumors: systematic review and meta-analysis. Ultrasound Obstet Gynecol 2015;46:277-83.

28 Liu Z, Yang F, Zhang Y, et al. Conventional, Doppler and contrastenhanced ultrasonography in differential diagnosis of ovarian masses. Cell Physiol Biochem 2016;39:2398-408.

29 Medeiros LR, Freitas LB, Rosa DD, et al. Accuracy of magnetic resonance imaging in ovarian tumor: a systematic quantitative review. Am J Obstet Gynecol 2011;204:67.e1-67.e10.

30 Byun JM, Kim YN, Jeong DH, et al. Three-dimensional transvaginal ultrasonography for locally advanced cervical cancer. Int $J$ Gynecol Cancer 2013;23:1459-64. 
31 Valentin L. Gray scale sonography, subjective evaluation of the color Doppler image and measurement of blood flow velocity for distinguishing benign and malignant tumors of suspected adnexal origin. Eur J Obstet Gynecol Reprod Biol 1997;72:63-72.

32 Van Calster B, Timmerman D, Bourne T, et al. Discrimination between benign and malignant adnexal masses by specialist ultrasound examination versus serum CA-125. J Natl Cancer Inst 2007;99:1706-14.

33 Testa AC, Timmerman D, Van Belle V, et al. Intravenous contrast ultrasound examination using contrast-tuned imaging $(\mathrm{CnTl})$ and the contrast medium SonoVue for discrimination between benign and malignant adnexal masses with solid components. Ultrasound Obstet Gynecol 2009;34:699-710.

34 Huchon C, Metzger U, Bats A-S, et al. Value of three-dimensional contrast-enhanced power Doppler ultrasound for characterizing adnexal masses. J Obstet Gynaecol Res 2012;38:832-40.

35 Veyer L, Marret $\mathrm{H}$, Bleuzen A, et al. Preoperative diagnosis of ovarian tumors using pelvic contrast-enhanced sonography. $J$ Ultrasound Med 2010;29:1041-9. 